\title{
The Effects of Virtual Reality on the Upper Extremity Skills of Girls with Rett Syndrome: A Single Case Study
}

\author{
Kourtney Mraz ${ }^{1, *}$, Grace Eisenberg ${ }^{1}$, Pamela Diener ${ }^{2}$, Gina Amadio ${ }^{1}$, Matthew H. Foreman ${ }^{1}$ \\ and Jack R. Engsberg ${ }^{1}$
}

${ }^{1}$ Washington University in St. Louis-School of Medicine, Program in Occupational Therapy, 4444 Forest Park Ave, Saint Louis, MO 63018, USA

${ }^{2}$ Georgetown University-School of Medicine, Department of Neuroscience, 3970 Reservoir Rd, NW, Washington, DC 20057, USA

\begin{abstract}
Introduction: Rett Syndrome (RTT) is a genetic disorder primarily seen in females that inhibits the use of a girl's hands in everyday activities. A girl with RTT spends the majority of her day engaged in stereotypical hand wringing/mouthing movements at midline of the body. The probable cause behind the neurological effects of RTT is a mutation in the gene that encodes for methyl-CpG protein 2 (MeCP2). The hand wringing/mouthing behaviors preclude a girl with RTT from using the upper extremities in purposeful tasks such as school work, play skills, and other activities of daily living.

Objectives: To develop a virtual reality (VR)-based therapeutic intervention that 1) decreases upper extremity stereotypies (repetitive movements that serve no function) that interfere with purposeful arm and hand use and 2) promotes purposeful, goal-directed arm function; improve upper extremity motor skills in girls with RTT.

Materials and Methods: Using FAAST Software and Microsoft Kinect sensor, one girl with RTT participated in a 12-week IVR intervention (1 hour/session, 3 sessions/week, 36 total hours). Pre- and post-assessments were administered to examine any changes in upper extremity function.

Results: The VR intervention led to improvements in use of the upper extremities to complete self-care activities, an increased number of reaches completed in a 15-minute period, and decreased time engaged in stereotypical hand movements.

Conclusion: Future work will add additional support to determine the effectiveness of virtual reality as an intervention for girls with RTT.
\end{abstract}

Keywords: Rett Syndrome, internet-based virtual reality, Microsoft Kinect, upper extremity movements, upper extremity stereotypies.

\section{INTRODUCTION}

Rett Syndrome (RTT) is second only to Down Syndrome as the primary source of neurological dysfunction in females. RTT is a genetic disorder almost exclusively in females that affects the use of a girl's hands in everyday living [1, 2]. A girl with RTT spends the majority of her day engaged in stereotypical hand wringing/mouthing movements at midline of the body (hands clasped together or at mouth). The presumed cause for the neurological effects of RTT is a mutation in the gene that encodes for the methyl-CpG protein 2 (MeCP2). This protein plays a role in the development of mature neurons; when development of these neurons is inhibited, gait and motor abnormalities can occur. MeCP2 deficits can also lead to changes in cortical excitation and inhibition, which has been associated with the upper extremity stereotypies seen in girls with RTT [3]. These behaviors preclude her from using the upper extremities in purposeful tasks

*Address correspondence to this author at 5837 Nina Place, St. Louis, MO 63112, USA; Tel: 815-450-0069; Fax: 314-286-1601;

E-mail: kmraz44@gmail.com such as school work, play skills, self-feeding, and other activities of daily living [1, 2].

Worldwide, RTT has a prevalence rate of 1 in 10,000 live female births. It is characterized by normal birth and psychomotor development until between 6 18 months of age, during which time regression in language and motor skills occurs [2]. Over time, girls with RTT develop a variety of disabilities, including loss of functional hand use and acquired speech, ataxia (uncoordinated movements), apraxia (difficulty initiating movements), and muscle tone irregularities. Current treatment for RTT includes interventions such as physical, occupational, hippo-, aquatic, and music therapies aiming to minimize decline in functional arm movements or promote improvement in skilled function (i.e. feeding and play skills). Evidence to support the proper dosing or efficacy of these interventions individually or in combination is limited. Consequently, best practice for treatment remains unknown [1, 2, 4].

Current literature in neuroscience and rehabilitation support the idea that challenging movement training is 
essential to bring about lasting changes in motor system networks, motor learning, and motor function. Repetitive challenging movements stimulate the molecular pathways of learning and memory within the motor system [5]. As a result, specific neuronal connections are potentiated, facilitating motor system connectivity $[6,7]$. These principles can be used to achieve large numbers of challenging movement repetitions that are therapist-directed, motivational and engaging, and provide quantitative feedback with the purpose of improving self-initiated use of the upper extremities for functional activities. An effective way to increase motivation for therapy and enhance the frequency, duration, and intensity of therapy movements is the use of VR and gaming therapy [8, 9]. The motivating nature of VR interventions can lead to completion of 300-600 movement repetitions in a single 40-minute session $[2,10]$.

It is clear that VR can be used to create a motivating intervention that will provide the repetition needed to facilitate motor training in girls with RTT. The utilization of VR can be used to potentially address the stereotypical hand wringing/mouthing movements seen in girls with RTT. The purpose of this project was to decrease hand wringing/mouthing and increase the use of the upper extremities in purposeful activities in a girl with RTT using VR therapy.

\section{METHODS}

\section{Subject}

One participant was recruited through a neurologist from St. Louis Children's Hospital, also currently working at the Rett Syndrome Clinic at Washington University in St. Louis. The inclusion criteria included: use of eyes to scan near and far, upper extremity functional range of motion, attention to tasks (with breaks) for 60-90 minutes, and an understanding of cause and effect. The understanding of cause and effect is crucial for participants to be able to connect movement of hand/arm with an in-game response. Informed consent was obtained from the mother before pre-intervention assessment began. The study was approved by the Washington University Medical Institutional Review Board. The participant was a nineyear-old female diagnosed with Rett Syndrome. She used an augmentative communication device to communicate. The upper extremity stereotypies of this participant manifested in the form of hair twirling and hand mouthing.

\section{Device Description}

The Microsoft Kinect sensor along with FAAST software was used in the intervention to permit upper extremity movements to control the VR activities [11, 12]. The Kinect sensor is a motion sensing input device that allows the user to control and interact with the game without touching a controller, wearing markers, or manipulating some other input device. The Kinect is able to track the orientation and position of $15-23$ joints in real-time.

The FAAST software permitted the mapping of a body movement (input) to a key stroke or mouse movement (output) [13, 12]. The desired movement came from a predefined library of actions. The therapist then defined the magnitude of the action to elicit a computer response. The keyboard output also came from a predefined list of virtual events. Using these predefined actions and virtual events permitted the setting of the controls (e.g. when right hand is above right wrist by at least $10 \mathrm{~cm}$, press up arrow key). The ability to translate the movements into keystrokes or mouse movements is what allows conversion of almost any VR game or YouTube video to become personalized therapy (Figure 1).

A video camera was used to record participants playing the games for later analysis. An HD-TV and HDMI cord (connected to the TV) were used permitting games to be seen on a larger scale.

\section{Intervention}

The participant completed a VR intervention 3 times per week for 12 weeks, each session lasting 60 minutes. The participant lived approximately 2 hours from the Human Performance Laboratory, where a

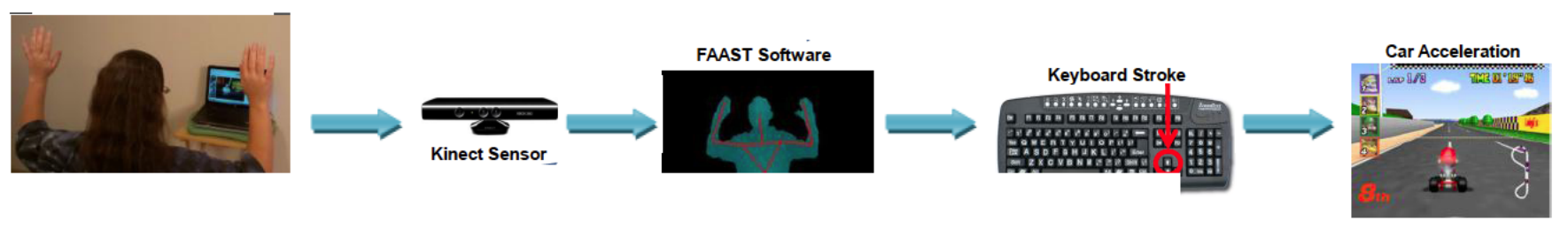

Figure 1: Process for converting participant's movements to VR activity. 
portion of the intervention was originally designed to take place. The family felt they could not travel this distance 3 days per week, which led to all treatment sessions taking place at the participant's home. The family was contacted biweekly to ensure that the intervention was proceeding smoothly.

The primary investigators administered the VR intervention for the first 2 sessions, while educating the primary caregiver in the process. The caregiver was provided with a handout detailing step-by-step instructions for how to operate the devices and software required to complete the intervention. The intervention was phased into being completely administered by the caregiver; for the first week, the caregiver administered the intervention once, twice in the second week, and completely independently by the third week. The primary investigators visited the home at weeks 4 and 10 to ensure that the intervention was proceeding without difficulty. The sessions the primary investigators attended were recorded using a video camera for later review.

In the first session, the targeted movement was set as forward reaching, requiring elbow extension and shoulder flexion/extension of the right arm. The movement threshold was set based on the participant's range of motion. YouTube videos used during the intervention were chosen by the participant. These videos included Frozen sing-along songs, nursery rhymes, music videos, and other Disney videos. Verbal cues and encouragement were consistently provided to initially train the participant to successfully participate in the intervention, but were decreased and only used as needed. The participant was required to reach forward in order to cause the YouTube video to play. After approximately 10-15 seconds, the primary investigator or the caregiver would pause the video, requiring the participant to reach forward to play the video again. Once a video was completed, the participant would point to the next video she wanted to play.

As the participant progressed throughout the intervention, the target movement was addressed to keep the activity a challenge. The forward reaching target was kept the same, but the arm required for the task was switched to the left, forcing the participant to use her other upper extremity. At 10 weeks, the target movement was then changed to forward reaching with either arm, allowing the participant to reach forward with both arms in order to cause the YouTube video to play. Although the threshold was not changed throughout the intervention, changing which arm was targeted provided a level of challenge for the participant and forced her to use both extremities throughout the intervention.

\section{Assessments}

To determine any effects of VR in improving the functional use of the upper extremities, assessments were administered. The assessments included: qualitative parent interviews, the Functional Reach Test, Goal Attainment Scaling, and the Pediatric Evaluation of Disabilities Inventory. These assessments were completed 1 week prior to the intervention and 1 week post-intervention. Two gift cards were provided to the parent, 1 prior to the intervention and 1 upon completion.

The Functional Reach Test is a measure that examines functional use of the upper extremities. This methodology was modified from a previous paper [14]. In brief, the test involves recording the time needed to self-initiate separation of hands and the distance reached to obtain a preferred item or engage a single switch to activate a battery-operated toy. A video camera is positioned in front of subject and tester to allow recording of the number of times the subject reaches for the item/switch, the number of times the subject removed her hand from her mouth or stopped wringing her hands, and the amount of time she spends not hand wringing/mouthing. The number of reaches and the time spent with hands separated is evaluated in a 15-minute time period. The distance reached is measured using a standard tape measure. The quality of movement is also examined on a scale of 0 (lack of upper extremity movement) to 5 (upper extremity movement resulting in contact with the preferred item) [14].

The Goal Attainment Scale (GAS) captures functional changes in an individual's performance by examining achievement of goals. Interviews are conducted with clients to collaboratively set goals, predict graded levels of possible goal attainment, and descriptions of expected performance for each goal following intervention. Scoring is done on a 5-point scale ranging from most favorable $(+2)$ to least favorable (-2) outcomes (Table 1) [15]. The GAS has good inter-rater reliability $(0.89)$ as determined by a systematic review [16]. No training is required for the GAS, but researchers should become familiar with using the scale prior to administration. GAS was used to provide more quantitative information on functional changes. Parents were involved in creating the goal 
and provided information to determine if the goal was met. Level of goal attainment can indicate any changes in activity participation and quality of life.

Table 1: Graded Levels of GAS

\begin{tabular}{|c|c|}
\hline Rating & Level Description \\
\hline \hline-2 & Least favorable outcome \\
\hline-1 & Less than expected outcome \\
\hline 0 & Expected outcome \\
\hline+1 & More than expected outcome \\
\hline+2 & Most favorable outcome \\
\hline
\end{tabular}

The Pediatric Evaluation of Disability Inventory (PEDI) is a measure of the functional ability of children ages 6 months to 7.5 years, although it can also be used with older children if their functional abilities are below those of a 7-year-old without disabilities. It examines the functional levels of the child and the amount of caregiver assistance required to complete daily activities. The PEDI has 3 domains: self-care, mobility, and social function; there is a functional skills section that examines if a child is unable (0) or capable (1) of completing an activity. There is also a caregiver assistance portion that assesses the level of assistance required to complete daily activities and is scored on a scale of 0 (maximal assistance) to 5 (independent), with higher scores indicating greater independence in daily activities. The PEDI is administered to the primary caregiver whose responses are based on typical daily interactions with the child. The raw scores for the items in a domain are totaled into a summary score for each of the domains. Interrater reliability $(0.95-0.99)$, intrarater reliability $(0.84-1.00)$, content validity $(0.80$ - 0.97), and concurrent validity $(0.80-0.92)$ were found to be high for this assessment [17]. One study found that a score increase of $11 \%$, approximately an 11-point increase, represents minimal clinically important difference [18].

\section{Data Analysis}

The qualitative parent interviews were used to examine changes noticed by the caregiver after completing the treatment sessions and gain insight into the ease of technology use. Pre-intervention answers and post-intervention answers were compared to determine changes observed as well as attitude towards the intervention and technology. The GAS was analyzed through caregiver report. The individualized goal and expected outcomes were determined in the initial interview with the caregiver, along with expected outcomes. The score was standardized by writing goals that had responses theoretically spaced the same distance apart. With this, a mathematical method can be derived through calculating the extent to which the goal is met. However, because there was just one goal established, only the scaled rating was determined.

The PEDI was analyzed by examining changes in the raw scores of the participant in each of the domains. Normative standard scores could not be used because the PEDI was standardized for children up to the age of 7.5 and the participant did not fit this age requirement. The Functional Reach Test was recorded using a video camera. The distance the participant reached for a preferred object was measured with a tape measure; the distance from the starting position of her hand to the object she reached for was measured. The time taken to initiate hand separation was measured with a stop watch and verified by watching the video and timing it again. The time spent with hands separated and the number of reaches was

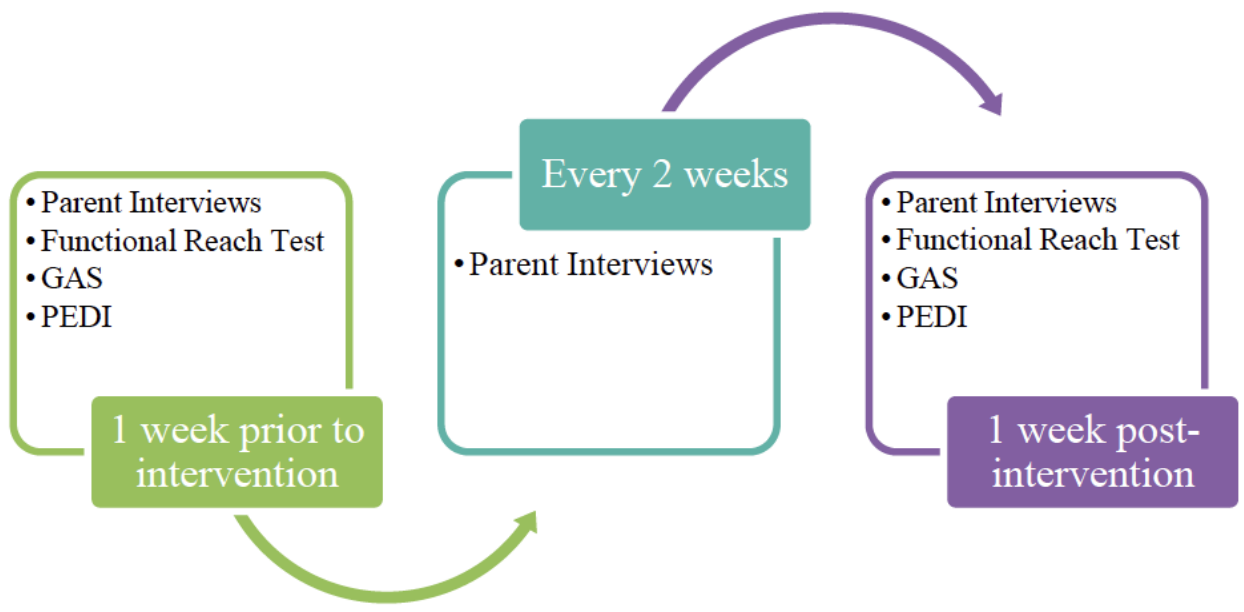

Figure 2: Sequence of events. 
determined by watching the video recording of the assessment. The quality of upper extremity movement was observed both during the assessment and verified by watching the recording. Video recordings during the intervention were examined to see if the Kinect was missing some of the participant's reaching movements.

\section{RESULTS}

\section{Parent Interviews}

Bi-weekly interviews provided information regarding the participant's progress and addressed any technical complications. The interviews revealed that the participant was enjoying the intervention and standing for all 60 minutes of the game play. Decrease in stereotypical hand movement was not noted until week 9 of intervention, with parent stating that she observed decrease in amount of time participant's hand were in her hair. Multiple interviews indicated that there were technological difficulties due to poor Internet access in home, in which the Kinect system had to be restarted.

The post-intervention parent interview indicated changes in the participant's upper extremity arm function due to less time spent with hands in hair. Additionally, the parent noted that there was an increase in use of hands for daily activities with an increase in using silverware for eating with fewer reminders from parent necessary. Parent indicated she was happy with the results of the intervention and that it was engaging and made the participant more tired. Parent noted that the participant was sleeping better at night due to increase of physical activity during game play. When asked about the ease of use of technology, the parent stated that using the Kinect and FAAST system were very simple and easy to use. Parent suggested that treatment sessions of 30-45 minutes may be easier due to busy home schedule.

\section{Functional Reach Test}

There was minimal change in the time to initiate hand separation. Pre-intervention the participant's time was 5.2 seconds; post-intervention her time was 5.8 seconds. The participant received a score of 5 (optimal upper extremity movement) both pre- and postintervention. The distance reached increased from 13 inches pre-intervention to 19.8 inches post-intervention. Changes in the number of reaches and the time spent with hands separated were dramatic from pre- to postintervention. Pre-intervention the participant kept her hands apart for 3.43 minutes out of the total 15 and completed 59 reaches in this time period. Postintervention the participant spent 8.91 minutes with her hands separated and completed 127 reaches. Minimal clinical important difference has not yet been established for the Functional Reach Test.

\section{Goal Attainment Scaling (GAS)}

Based on the goal created prior to the intervention, participant's overall performance following the intervention was much better than the expected level of performance. Pre-intervention the participant kept one or both hands in either her hair or mouth for $75 \%$ of her time at home. Post-intervention the caregiver indicated that the amount of time with the participant's hands in her hair or mouth had decreased to $45-50 \%$ of her time at home. The most favorable outcome was observed, with a rating of +2 .

\section{Pediatric Evaluation of Disability Inventory (PEDI)}

There was a clinically important difference in the self-care domain of the functional skills portion [18]. Pre-intervention the participant received a raw score of 28; post-intervention she received a score of 40 . Improvements in upper extremity use for functional activities were primarily seen in participation in using utensils while eating, self-feeding, hygiene, dressing, and toileting. Changes in the raw scores pre- and postintervention were minimal for the mobility or social function domains in the functional skills portion of the PEDI or for any of the domains in the caregiver assistance portion.

Table 2: Functional Reach Test Results

\begin{tabular}{|c|c|c|}
\hline & Pre & Post \\
\hline \hline Time to Self-Initiate Hand Separation & 5.2 seconds & 5.8 seconds \\
\hline Distance Reached & 13 inches & 19.8 inches \\
\hline Time Spent with Hands Separated & 3.43 minutes & 8.91 minutes \\
\hline Number of Reaches & 59 & 127 \\
\hline
\end{tabular}


Table 3: Goal Attainment Scaling Goal for Participant

Concern: Inability to independently participate in daily activities due to limited upper extremity movement with hands constantly in hair or mouth $75 \%$ of time at home.

Goal: To be able to participate in daily activities by decreasing amount of time of hands in hair or mouth to only $60 \%$ of time at home.

Intervention Period: 36 sessions

\begin{tabular}{|c|c|c|c|c|}
\hline $\begin{array}{c}\text { Much Less Than } \\
\text { Expected Level }\end{array}$ & $\begin{array}{c}-1 \\
\text { Less Than } \\
\text { Expected Level }\end{array}$ & $\begin{array}{c}0 \\
\text { Expected Level of } \\
\text { Performance }\end{array}$ & $\begin{array}{c}+1 \\
\text { Better Than Expected } \\
\text { Level }\end{array}$ & $\begin{array}{c}\text { Much Better Than } \\
\text { Expected Level }\end{array}$ \\
\hline $\begin{array}{c}\text { Hands in hair or mouth } \\
70 \% \text { of time at home }\end{array}$ & $\begin{array}{c}\text { Hands in hair or mouth } \\
65 \% \text { of time at home }\end{array}$ & $\begin{array}{c}\text { Hands in hair or mouth } \\
60 \% \text { of time at home }\end{array}$ & $\begin{array}{c}\text { Hands in hair or mouth } \\
55 \% \text { of time at home }\end{array}$ & $\begin{array}{c}\text { Hands in hair or mouth } \\
50 \% \text { of time at home }\end{array}$ \\
\hline
\end{tabular}

*Indicates observed outcome post-intervention.

Table 4: PEDI Results

\begin{tabular}{|c|c|c|c|}
\hline & Pre & Post & Change \\
\hline Self-Care - Functional Skills & 28 & 40 & 12 \\
\hline Mobility - Functional Skills & 58 & 56 & -2 \\
\hline Social Function - Functional Skills & 39 & 36 & -3 \\
\hline Self-Care - Caregiver Assistance & 8 & 8 & 0 \\
\hline Mobility - Caregiver Assistance & 33 & 31 & -2 \\
\hline Social Function - Caregiver Assistance & 7 & 11 & 4 \\
\hline
\end{tabular}

\section{DISCUSSION}

The purpose of this investigation was to increase the use of the upper extremities for purposeful activities and decrease hand wringing/mouthing using VR as a therapeutic medium. While this single case study did yield promising results, there were several limitations. The participant's caregiver stated that they did not maintain $100 \%$ compliance with the VR intervention due to issues with their Internet connection. This lack of compliance with the intervention may have negatively affected the impact it had on the participant. Video recordings of the intervention indicated that the Kinect did not pick up all of the movements completed by the participant, demonstrating a limitation with the technology used in the intervention. The Functional Reach Test was designed for individuals with a lower cognitive ability and slower processing time than the participant's. For this reason, it may not have been the most appropriate assessment for this participant. The Functional Reach Test calls for a training of cause and effect, which was already well-established in this participant. As it was designed for individuals with fairly significant cognitive deficits, it calls for repetitive movement toward one target, which is likely not challenging or motivating for individuals with higher cognitive functioning. It may be more useful to examine the time spent with hands separated and the number of reaches during 15-minute portions of the first and last intervention sessions. Additionally, post-intervention, the participant demonstrated more difficulty reaching for the item because she had become so proficient at the intervention that she was initially attempting to just complete a reaching motion instead of actually making contact with the preferred item.

This single case study provides support for the use of $V R$ as an intervention for girls with RTT. While further work in this area is needed, the results from this study were promising. Improvements were seen in the purposeful use of the upper extremities for daily activities and in the amount of time spent in upper extremity stereotypies. This change can be examined through the lens provided in the literature on neuroscience and rehabilitation; as literature has shown, motor function changes are facilitated by the repetitive, challenging movements that were provided by this VR intervention [5, 8, 9]. The parent reported high satisfaction with the intervention and provided qualitative information on improvements she had seen in her daughter. The findings from the self-care, functional skills domain of the PEDI demonstrated minimum clinical important difference. Improvements in upper extremity use were also noted during the Functional Reach Test, but a minimum clinical important difference has not yet been established. It is 
possible that the participant's understanding of cause and effect may be linked to previous experience with technology-related research studies and a higher cognitive level, allowing for transfer of skills and knowledge. Hydrotherapy has been effective in decreasing hand stereotypies and increasing functional hand use, but is not as flexible a medium of therapeutic intervention as VR [19]. Additionally, hand splints were found to lead to some improvements in hand function, but results were inconsistent and some individuals with RTT are unable to tolerate the splints [20].

Future research with a larger sample size is needed to establish the effects of VR on the upper extremity use of individuals with RTT. As best practice for treatment of individuals with RTT is still unknown $[1,2$, 4], future studies could examine the proper dosing and efficacy of VR interventions to enrich the knowledge on best practice. The current study can be used to inform the protocol for future studies. Future research could be conducted to determine the quantity of the VR intervention needed to achieve maximal motor performance. Additionally, a future study could use a VR intervention to address the deficits in cognition and ambulation that are present in individuals with RTT. VR could be used in a clinical setting to provide a motivating intervention that could be transferred into the home setting; results pertaining to usability of the intervention indicate that it is feasible for parents to implement it independently in the home. The long-term effects of this intervention could be examined in future studies. VR may have the potential to be very successful in improving the quality of life of individuals with RTT, necessitating further research in this area to establish it as an effective intervention.

The purpose of this project was to decrease hand wringing/mouthing and increase the use of the upper extremities in purposeful activities using VR therapy. The participant showed improvements in time spent with hands in hair or mouth, and an increase in upper extremity use for functional activities such as using utensils while eating, self-feeding, hygiene, and dressing. The caregiver also indicated the participant was sleeping better at night due to increase of physical activity during game play. The results from this study suggest that VR therapy may be a valid tool to be used with the RTT population and may decrease stereotypical hand movements and increase functional hand movements. Improvement in this area may contribute to improved quality of life and increased participation in activities. Further research on the use of VR with individuals with RTT is needed to support its use as an effective treatment approach with this population.

\section{REFERENCES}

[1] Lotan M, Hanks S. Physical therapy intervention for individuals with Rett syndrome. Sci World J 2006; 6: 131438.

http://dx.doi.org/10.1100/tsw.2006.187

[2] Lotan M. Rett Syndrome: guidelines for individual intervention. Sci World J 2006; 6: 1504-16.

http://dx.doi.org/10.1100/tsw.2006.252

[3] Cuddapah VA, Nwaobi SE, Percy AK, Olsen ML. MECP2 in the regulation of neural activity: Rett syndrome pathophysiological perspectives. Degener Neurol Neuromuscul Dis 2015; 5: 103-16.

[4] Lin J, Kelleher CL, Engsberg JR. Developing home-based virtual reality therapy interventions. Games Health 2013; 2(1): 34-8

http://dx.doi.org/10.1089/g4h.2012.0033

[5] Adkins DL, Boychuk J, Remple MS, Kleim JA. Motor training induces experience-specific patterns of plasticity across motor cortex and spinal cord. J Appl Physiol 2006; 101: 1776-82. http://dx.doi.org/10.1152/japplphysiol.00515.2006

[6] Carmichael ST. Themes and strategies for studying the biology of stroke recovery in the poststroke epoch. Stroke 2008; 39: 1380-88.

http://dx.doi.org/10.1161/STROKEAHA.107.499962

[7] Nudo RJ, Plautz EJ, Frost SB. Role of adaptive plasticity in recovery of function after damage to motor cortex. Muscle Nerve 2001; 24: 1000-19. http://dx.doi.org/10.1002/mus.1104

[8] Sevick, MR, Mensch AR, Foreman MH, Standeven JW Engsberg JR. Feasibility and effects of using internet virtual reality based gaming therapy: improving upper extremity functioning for children with cerebral palsy. Behavioral Sciences 2016; 6: 10. http://dx.doi.org/10.3390/bs6020010

[9] Chuang YC. Massively multiplayer online role-playing gameinduced seizures: a neglected health problem in Internet addiction. Cyberpsychol Behav 2006; 9(4): 451-6. http://dx.doi.org/10.1089/cpb.2006.9.451

[10] Lauterbach SA, Foreman MH, Engsberg JR. Computer games as therapy for persons with stroke. Games Health 2013; 2(1): 24-8.

http://dx.doi.org/10.1089/g4h.2012.0032

[11] Huber M, Rabin B, Docan C, Burdea GC, AbdelBaky M, Golomb MR. Feasibility of modified remotely monitored inhome gaming technology for improving hand function in adolescents with cerebral palsy. IEEE Trans Infor Technology Biomed 2010; 14(2): 526-34. http://dx.doi.org/10.1109/TITB.2009.2038995

[12] Naidu S. Rett syndrome. Indian J Pediatr 1997; 64: 651-9. http://dx.doi.org/10.1007/BF02726119

[13] International Rett syndrome Foundation. About Rett syndrome. http://www.rettsyndrome.org/about-rett-syndrome.

[14] Himmelrich M, Barnes L, Heinz V, Manetti J, Myers M, Sant G, Crabtree I, Ridpath A, Diener P (in preparation). Modified sensory integration therapy minimizes regression and improves postural control and UE use in a girl with Rett syndrome: a single subject design.

[15] Cusick A, McIntyre S, Novak I, Lannin N, Lowe K. A comparison of goal attainment scaling and the Canadian occupational performance measure for paediatric rehabilitation research. Ped Rehab 2006; 9(2): 149-57. http://dx.doi.org/10.1080/13638490500235581 
[16] University of Illinois at Chicago: virtual reality's CAVE pioneer. http://www.edtechmagazine.com/higher/article/2013/ 01/university-illinois-chicago-virtual-realitys-cave-pioneer August 13, 2013.

[17] Nichols, DS, Case-Smith, J. Reliability and validity of the Pediatric Evaluation of Disability Inventory. Pedatr Phys Ther 1996; 8: 15-24. http://dx.doi.org/10.1097/00001577-199600810-00004

[18] lyer, LV, Haley SM, Watkins, MP, Dumas, HM. Establishing minimal clinically important differences for scores on the
Pediatric Evaluation of Disability Inventory for inpatient rehabilitation. Phys Ther 2003; 83(10): 888-98.

[19] Buman G, Uyanik M, Yilmaz I, Kayihan H, Topcu M. Hydrotherapy for Rett syndrome. J Rehabil Med 2003; 35: 44-5.

http://dx.doi.org/10.1080/16501970306107

[20] Van Acker R. Rett syndrome: a review of current knowledge. J Autism Dev Disord 1991; 21(4): 381-406. http://dx.doi.org/10.1007/BF02206866

\section{DOI: http://dx.doi.org/10.6000/2292-2598.2016.04.03.2}

(C) 2016 Mraz et al.; Licensee Lifescience Global.

This is an open access article licensed under the terms of the Creative Commons Attribution Non-Commercial License (http://creativecommons.org/licenses/by-nc/3.0/) which permits unrestricted, non-commercial use, distribution and reproduction in any medium, provided the work is properly cited. 